\title{
THE AUTOMORPHISMS OF THE SYMMETRIC GROUP
}

\author{
IRVING E. SEGAL
}

The purpose of this note is to give a proof of the following well known theorem. The group of automorphisms of the symmetric group $S_{n}$ on $n$ letters is isomorphic with $S_{n}$, except when $n=6$. The proofs of this in the literature are complicated ${ }^{1}$ and involve the use of lemmas whose relevance is not plain.

Let $A$ be an automorphism of $S_{n}$. Then it is clear that $A$ takes a class of similar elements into a class of similar elements, and that it takes an element of order $m$ into an element with the same order. Hence suppose $A(1 r)=t_{1}(r) \cdot t_{2}(r) \cdots \cdots t_{k}(r)(k \geqq 1)$, where the $t_{i}(r)$ are disjoint transpositions. A simple calculation shows that there are $n(n-1) / 2$ elements similar to $(1 r)$, and that there are $n ! / 2^{k} k !(n-2 k)$ ! elements similar to $t_{1}(r) \cdot t_{2}(r) \cdots \cdot t_{k}(r)$. Hence

$$
\frac{n(n-1)}{2}=\frac{n !}{2^{k} k !(n-2 k) !} \text {. }
$$

If $n \neq 6$ this equation is satisfied for no $k(k \geqq 1)$ except $k=1$.

Suppose now that $n \neq 6$. Then $A(1 r)=\left(a_{r} b_{r}\right)$ say. If $r \neq 2,(12)(1 r)$ $=(12 r)$ (multiplying from right to left), and evidently, $A(12 r)$ $=\left(a_{2} b_{2}\right)\left(a_{r} b_{r}\right)$. Since $(12 r)$ has the order 3 , so has $\left(a_{2} b_{2}\right)\left(a_{r} b_{r}\right)$ and the transpositions $\left(a_{2} b_{2}\right)$ and $\left(a_{r} b_{r}\right)$ must have a letter in common. Then it is no loss to assume $a_{2}=a_{r}$ or $b_{2}=b_{r}$. However, if $a_{2}=a_{r}$ and $b_{2}=b_{s}$ $(r \neq 2, \quad s \neq 2)$, then $r \neq s$ and $A(12 r)=A(12) \cdot A(1 r)=\left(a_{2} b_{2}\right)\left(a_{2} b_{r}\right)$ $=\left(b_{r} a_{2} b_{2}\right)$. Similarly $A(12 s)=\left(a_{s} b_{2} a_{2}\right)$. Hence $A((12 r) \cdot(12 s))=A(12 r)$ $\cdot A(12 s)=\left(b_{r} a_{2} b_{2}\right)\left(a_{s} b_{2} a_{2}\right)=\left(b_{r} a_{s} b_{2}\right)$ which is of order 3 , while $(12 r)$ $\cdot(12 s)=(1 s)(2 r)$, which is of order 2 . Hence one must have $a_{2}=a_{r}$ for all $r$ or $b_{2}=b_{r}$ for all $r$; of course one can let $a_{2}=a_{r}(r=2,3, \cdots, n)$. Then $A(1 r)=\left(a_{2} b_{r}\right)$. Hence $A$ is precisely the automorphism $A$ defined by $A x=t^{-1} x t$, where

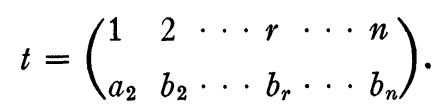

For $A x=t^{-1} x t$ when $x=(1 r)$, and the elements $\{(1 r)\}(r=2,3, \cdots, n)$ generate $S_{n}$.

Yale University

${ }^{1}$ The first proof is by O. Hölder, Mathematische Annalen, vol. 46 (1895), especially pp. 340-345. 